\title{
Modelagem de Usuários Baseada em Estilo de Aprendizagem, Teoria da Resposta ao Item e Lógica Fuzzy para Sistemas Adaptativos Educacionais
}

\author{
Rodrigo E. Carneiro ${ }^{1}$, Fernando B. Lima Neto ${ }^{1}$, Denis S. Silveira ${ }^{2}$ \\ ${ }^{1}$ Escola Politécnica de Pernambuco - Universidade de Pernambuco (UPE) \\ Recife - PE - Brasil \\ ${ }^{2}$ Centro de Ciências Sociais Aplicada \\ Universidade Federal de Pernambuco (UFPE) - Recife, PE - Brasil \\ $\{r e c, f b l n\} @ e c o m p . p o l i . b r, d s i l v e i r a @ u f p e . b r$
}

\begin{abstract}
The use of adaptive systems with educational objective represent an advance in relation to the traditional systems of tutorial, they provide a personalized instruction, overcoming some of the more critical problems of the current educative software. In this paper, we propose a modelling mechanism of cognitive profile using Fuzzy Logic and Item Response Theory combined with Learning Styles to personalize online trainings to the pace of each apprentice. The results obtained show that the proposed model propitiated a significant improvement in the development of the apprentices and got a bigger acceptation when valued qualitatively.
\end{abstract}

Resumo.O uso de sistemas adaptativos com objetivo educacional representa um avanço em relação aos sistemas de tutoria tradicionais, pois proporciona uma instrução personalizada, superando assim, alguns dos problemas mais críticos dos atuais softwares educativos. Neste artigo é proposto um mecanismo de modelagem de perfil cognitivo utilizando lógica fuzzy e teoria da resposta ao item. Isso, combinado ao uso de estilo de aprendizagem para personalização de treinamentos online ao ritmo de cada aluno, propicia resultados com melhorias significativas no desempenho dos aprendizes e grande aceitação quando avaliado qualitativamente.

\section{Introdução}

Atualmente, o Ensino a Distância (EAD) se tornou uma importante fonte de obtenção de conhecimento, fornecendo conteúdo sem a limitação de tempo ou espaços físicos. $\mathrm{O}$ uso da Internet para educação vem crescendo a cada ano, tanto para novos alunos quanto para ensino continuado de professores [Brasil, 2012].

Devido à grande variedade de perfis de usuários participantes desses cursos, torna-se cada vez mais difícil manter a qualidade e satisfação dos alunos com o modelo tradicional de ensino, já que o mesmo conteúdo é exibido para todos os participantes independentemente de suas idiossincrasias [Dias, Gasparini, \& Kemczinski, 2009; Schnitman, 2010].

Visando resolver esse problema, o presente artigo propõe o uso dos sistemas hipermídia adaptativos, que combinam ideias dos sistemas hipermídia e dos sistemas tutores inteligentes [Brusilovsky, 2001]. Ou seja, sistemas que sabem o que ensinar 
(conteúdo), para quem ensinar (modelagem do aluno) e como ensinar (estratégias pedagógicas ou de ensino)[Silva, 2000].

Várias pesquisas [Kavitha, Vijaya, \& Saraswathi, 2012][Mohamed, Bensebaa, \& Trigano, 2012][Bueno, Brito, \& Brito, 2011] foram feitas utilizando a Teoria da resposta ao item em sistemas adaptativos considerando as diferenças individuais dos aprendizes. Sobre Estilos de aprendizagem, a pesquisa de [Valaski, Malucelli, \& Reinehr, 2011] relata os principais modelos aplicados atualmente.

Neste trabalho é proposto um modelo que utiliza Estilos de Aprendizagem (EA), para a adaptação do conteúdo as características do aprendiz, além de utilizar Teoria da Resposta ao Item (TRI), Lógica Fuzzy e Rede Bayesiana, para gerenciar a sequência do treinamento em um sistema hipermídia adaptativo, com o objetivo de melhorar a representação computacional do conhecimento do aprendiz, o que representa uma melhora na qualidade da adaptação do treinamento.

\section{Fundamentação Teórica}

\subsection{Sistema hipermídia adaptativo}

Sistemas Hipermídia Adaptativos (SHA)[Brusilovsky, 2001], são sistemas capazes de se adaptar aos usuários. Quando usados com foco educacional, combinam ideias de sistemas hipermídia e sistemas tutores. Comumente, os SHAs são constituídos pelos seguintes componentes[Brusilovsky, 2001]:

- Modelo do Usuário (MU): componente responsável por representar o conhecimento do usuário do sistema. Essas informações são necessárias para a adaptação do conteúdo;

- Modelo de Adaptação (MA): responsável pela escolha das táticas e estratégias pedagógicas que serão aplicadas para o treinamento conforme os dados obtidos a partir do estado atual do MU;

- Modelo Domínio (MD): é o módulo especialista do sistema, responsável por gerenciar todo material instrucional que será apresentado ao aprendiz;

- Modelo Interface (MI): representa o mecanismo de interação entre o SHA e os usuários.

A partir da interação do usuário com o MI, é possível criar sua representação de conhecimento no MU, o que possibilita que os conteúdos do MD possam ser adaptados pelo MA. Portanto, o MU é fundamental para uma boa adaptação do sistema e por esse motivo é crescente alvo de estudo.

\subsection{Estilos de Aprendizagem}

Estudantes aprendem de muitas maneiras, como por exemplo, vendo e ouvindo; refletindo e atuando, entre outros. Essas formas ou estilos de aprendizagem podem ser representados por diversos modelos desenvolvidos por vários autores como Kolb[Kolb, 1974], Felder-Silverman[Felder \& Silverman, 1988], Fleming [Fleming, 2012], entre outros.

Cada autor utiliza diferentes dimensões para classificar as características dos aprendizes, por exemplo: 
- Felder-Silverman (1988): abrange quatro dimensões da aprendizagem. São elas: percepção (Sensorial/Intuitiva), entrada (Visual/Verbal), processamento (Ativo/Reflexivo) e entendimento (Sequencial/Global);

- Kolb (1973): é definido de acordo com duas dimensões. São elas: experiência concreta ou conceitualização abstrata e experimentação ativa ou observação reflexiva;

- VARK (1987): compreende quatro dimensões. São elas: visual, verbal, leitura/escrita e cinestésico.

Em [Valaski et al., 2011], identifica-se que o modelo mais referenciado no período de 2005 a 2011 foi o Felder-Silverman. Sendo por esse motivo o escolhido para aquisição do EA do aprendiz no sistema adaptativo proposto.

\subsection{Teoria da Resposta ao Item}

A Teoria da resposta ao item (TRI) é uma modelagem estatística usada geralmente para avaliação de habilidades e conhecimento em testes de múltiplas escolhas. Entre os modelos utilizados pela TRI, destaca-se o Modelo Logístico de 3 Parâmetros (ML3P), onde os itens são tratados de forma dicotômica (corrigidos como certo ou errado) [Andrade, Tavares, \& Valle, 2000].

No ML3P, cada questão é representada por uma curva característica e a probabilidade de um indivíduo com habilidade $\theta$ responder corretamente ao item é dada por (1):

$$
P_{i}(\theta)=P\left(U_{i}=1 \mid \theta\right)=c_{i}+\frac{1-c_{i}}{1+e^{-D a_{i}\left(\theta-b_{i}\right)}} .
$$

Na equação 1 , os coeficiente $b, a, c$ e $D$ representam, respectivamente, o grau de dificuldade, a discriminação, a probabilidade de acerto ao acaso e o fator de escala. Já a habilidade dos indivíduos é representada por $\theta[$ Spenassato \& Kinas, 2010]. Dessa forma, devido à natureza acumulativa do modelo, a probabilidade de um usuário responder corretamente um item cresce com o aumento de suas habilidades.

Na TRI, cada questão (item) tem seus parâmetros ( $a, b$ e $c$ ) estimados por meio de métodos estatísticos descritos e aceitos na literatura [Andrade et al., 2000; Baker, 2001]. E, com isso, a dificuldade do item e a habilidade do aprendiz passam a ser medidas com a mesma "régua". Por exemplo, um aprendiz com habilidade 1.5 terá maior probabilidade de acertar uma questão cuja dificuldade seja 1.2. Por essas razões, a TRI é hoje amplamente utilizada nos exames nacionais brasileiros promovidos pelo Ministério da Educação.

Como a TRI avalia a habilidade do usuário levando em consideração todas as suas respostas anteriores, é possível que dois aprendizes que tenham a mesma quantidade de acertos possuam pontuações diferentes. Por exemplo, em uma prova de dez questões, um aluno que acertou cinco, sendo a maioria questões fáceis, teria uma pontuação maior que um segundo aprendiz que acertou a mesma quantidade de questões, porém sendo a maioria questões difíceis. A incoerência pedagógica do segundo aprendiz é entendida pela TRI como um acerto ao acaso. Portanto, seu acerto não tem tanto valor quanto o primeiro. Diferente da Teoria Clássica dos Testes (TCT), a 
TRI não pergunta quantos itens o aprendiz acertou, e sim por que ele acertou ou errou cada item individualmente [Pasquali \& Primi, 2003].

\subsection{Lógica Fuzzy}

A lógica difusa (LD) é a lógica baseada na teoria dos conjuntos nebulosos (conjuntos fuzzy), formalizada pelo Prof. Lotfi Zadeh[Zadeh, 1965], em 1965, com o objetivo de manipular a incerteza da informação. Utiliza regras, compostas por variáveis linguísticas, o que torna mais intuitiva e realista a modelagem do sistema [Pedrycz, 1993]. Portanto, altamente alinhada com a demanda do objetivo desse trabalho.

Segundo Zadeh (1965), a função de pertinência de um elemento varia entre 0 e 1 e representa o quanto esse elemento pertence a um determinado conjunto, sendo 1 quando ele pertence completamente e 0 quando não pertence ao conjunto. Qualquer valor intermediário indica pertinência parcialmente compatível com o conjunto. Portanto, uma generalização à teoria dos conjuntos como foi proposta por Aristóteles.

Um conjunto $X$ da teoria dos conjuntos clássica pode ser entendido como um conjunto difuso específico, denominado usualmente de "crisp", para o qual $A \mu A$ : $\mathrm{U}\{0,1\}$, ou seja, a pertinência é do tipo "tudo ou nada", "sim ou não", e não gradual, como para os conjuntos fuzzy[Sandri, 1999].

A lógica difusa, devido às características aqui referenciadas, torna-se indicada em sistemas adaptativos, principalmente para servir de interface de representação do usuário, etapa imprescindível do mecanismo de adaptação.

\section{Modelo Proposto}

\subsection{Mecanismo fuzzy-TRI}

O tempo que o aprendiz utilizou para responder cada questão e sua dificuldade, são utilizados como entrada em um mecanismo fuzzy, que tem como objetivo penalizar respostas aleatórias.

Utilizando o parâmetro $b$ da ML3P de cada item, pode-se classificar uma questão em "Dificuldade Fácil (D_F)", "Dificuldade Médio (D_M)" e "Dificuldade Difícil (D_D)" com os conjuntos de pertinência. Para cada questão, é estimado o tempo médio de resposta e o desvio padrão. A partir desses dados, foram definidos conjuntos difusos representando o tempo de resposta e classificados em "Tempo Muito Rápido (T_MR)", “Tempo Rápido (T_R)", “Tempo Normal (T_N)", "Tempo Lento (T_L)" e "Tempo Muito Lento (T_ML)".

O controlador fuzzy proposto calcula o valor da penalização do aluno utilizando os conjuntos difusos de saída: "Penalização Não Penaliza (P_NP)", "Penalização Penaliza Pouco (P_PP)", "Penalização Penaliza (P_P)" e "Penalização Penaliza Muito (P_PM)" associados as regras fuzzy apresentadas na Tabela 1. 
Tabela 1. Regras fuzzy utilizadas.

\begin{tabular}{|c|c|c|c|c|c|}
\hline \multirow{2}{*}{ Dificuldade } & \multicolumn{5}{|c|}{ Tempo Resposta } \\
\cline { 2 - 6 } & $\boldsymbol{T}$-MR & $\boldsymbol{T} \_\boldsymbol{T}$ & $\boldsymbol{T} \_\boldsymbol{T}$ & $\boldsymbol{T} \boldsymbol{L}$ & $\boldsymbol{T} \boldsymbol{M L}$ \\
\hline D_F & P_PM & P_NP & P_NP & P_P & P_PM \\
\hline D_M & P_PM & P_P & P_NP & P_P & P_PM \\
\hline D_D & P_PM & P_P & P_NP & P_P & P_P \\
\hline
\end{tabular}

Portanto, um aprendiz que responde corretamente uma questão classificada como "difícil" em um tempo "rápido", terá o parâmetro c da ML3P, que corresponde à probabilidade de acerto ao acaso, acrescido da saída do mecanismo fuzzy. Como o cálculo da habilidade do aprendiz leva em consideração a probabilidade de acerto de cada item utilizando o ML3P, significa que o acerto da questão contribuirá menos para sua pontuação final. O mesmo acontece quando uma questão considerada "fácil" é respondida em um tempo "lento".

Com isso, o parâmetro c da ML3P, que geralmente é 1, sobre a quantidade de respostas do item é incrementado da saída do CBLD, onde o novo valor, Cfuzzy, é aplicado conforme a equação (2).

$$
P_{i}(\theta)=P_{i}\left(U_{i}=1 \mid \theta\right)=C f u z z y_{i}+\frac{1-C f u z z y_{i}}{1+e^{-D a_{i}\left(\theta-b_{i}\right)}},
$$

Por exemplo, um item cujo seu parâmetro $b$ é convertido na variável linguística "D_D" e o tempo de resposta convertido em "T_MR" dispara a seguinte regra do controlador fuzzy: IS "P_PM".

IF dificuldade IS “D_D” AND tempoResposta IS “T_MR” THEN penalização

Tal regra é analisada no processo de inferência fuzzy e os conjuntos fuzzy de saída são convertidos em valor numérico no processo de defuzzyficação. $\mathrm{O}$ valor obtido é somado ao parâmetro $c$ do item, aumentando a probabilidade de acerto ao acaso do item quando a resposta for incoerente.

\subsection{Estilo de Aprendizagem}

A aquisição do estilo de aprendizagem (EA) utilizou o questionário desenvolvido por Felder-Silverman[Felder \& Silverman, 1988], mas com um número reduzido de perguntas. Foi utilizado um conjunto com as 20 perguntas mais representativas do questionário Felder-Silverman. Para cada dimensão de aprendizado foram escolhidas apenas 5, das 11 questões disponíveis, e suas escolhas basearam-se, segundo [Graf, Viola, \& Kinshuk, 2006], em sua representatividade.

A redução de 44 para 20 questões diminui a acurácia do questionário, porém ainda consegue classificar de forma eficiente os EA. Tal modificação foi realizada com o objetivo de tornar mais rápido e menos cansativo o cadastramento de novos aprendizes. 


\subsection{Mecanismo Adaptativo}

Baseando-se na dimensão de entrada do EA de cada usuário e em seu desempenho durante o treinamento, o sistema utilizou, como MA, a roleta de probabilidade para decidir qual tipo de tela seria exibida ao aprendiz. Para o estilo de aprendizagem verbal foram apresentados imagens e textos, enquanto para o estilo visual foram apresentados os conteúdos multimídia. Ainda no MA, o sistema fez uso do mecanismo de adaptação criada em [Bueno et al., 2011], onde o valor do conhecimento do aprendiz é utilizado como entrada em uma rede bayesiana que decide qual será a próxima tela a ser apresentada. Na Figura 1, pode-se observar o fluxo adaptativo.

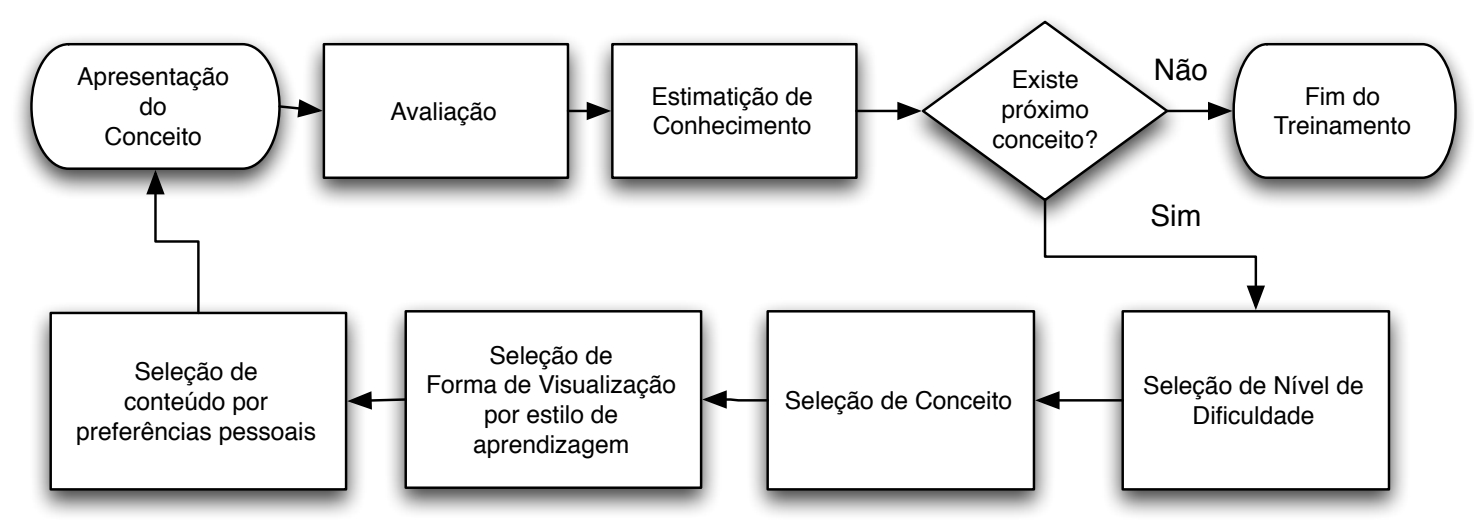

Figura 1. Fluxo da aplicação.

Após a apresentação de um novo conceito, o aprendiz é avaliado utilizando questionários baseados em TRI. O sistema utiliza o mecanismo fuzzy-TRI proposto para estimar o conhecimento do aprendiz sobre o conceito, e utiliza o modelo com rede bayesiana [Bueno et al., 2011] para decidir sobre a dificuldade e sequência das próximas telas a serem apresentadas.

\section{Aplicação Experimental}

Foi proposto um estudo empírico do tipo experimento voluntário, que tem como objetivo analisar quantitativamente e qualitativamente o uso da modelagem de usuário proposta nesse trabalho em um SHA.

Para o experimento foram utilizados conteúdos, MD, sobre inglês no nível A1. Para o MU, foi utilizado o modelo proposto que envolve TRI, lógica Fuzzy e estilo de aprendizagem. Para o MA foi utilizado o modelo proposto por [Bueno et al., 2011].

Após o treinamento no sistema adaptativo os aprendizes responderam um questionário qualitativo, com intuito de verificar sua opinião sobre o experimento e sugestões de melhorias, e um teste de 15 questões para avaliação quantitativa da ferramenta. Por se tratar de um sistema voltado para web, o acesso foi feito utilizando um navegador comum de Internet. O sistema foi implementado na linguagem PHP e o conteúdo e o MI desenvolvidos usando HTML e Adobe Flash. Para o armazenamento dos dados dos aprendizes foi utilizado o SGBD orientado a documento MongoDB 2.0. 


\section{Resultados}

Durante o experimento foram armazenados, de cada participante, seu cadastro pessoal, seu EA, suas respostas dos exercícios, seu questionário qualitativo e respostas do teste quantitativo. Como cadastro pessoal foi pedido nome, e-mail, data de nascimento e sexo, além das preferências.

O experimento contou com a participação de 56 aprendizes entre 11 e 59 anos sendo 28.1 a média de idade e 7.920 desvio padrão. Desses, $66 \%$ deles pertencia ao sexo masculino e $34 \%$ ao feminino, separados em 2 grupos. O primeiro grupo utilizou um sistema sem técnicas adaptativas, onde o mesmo conteúdo foi apresentado a todos os usuários, enquanto o segundo grupo fez uso do mecanismo adaptativo proposto nesse trabalho.

O estilo de aprendizagem mais frequente entre os participantes, na dimensão de Processamento, foi o Ativo, com $75 \%$ dos aprendizes encaixando-se nesta característica. $\mathrm{Na}$ dimensão Percepção, o estilo Sensorial obteve $90 \%$ do total de participantes. Na dimensão de Entrada, o estilo Visual foi o mais ocorrido com $82 \%$, e, por fim, na dimensão Organização, $51 \%$ ficaram com o estilo Global.

Apenas os usuários que afirmaram, no cadastro de inscrição, nunca ter participado de curso de inglês foram analisados quantitativamente, restando 30 aprendizes, sendo 15 do grupo 1 e 15 do grupo 2. Após o teste, os resultados dos dois grupos foram estatisticamente analisados. Utilizou-se o teste de Shapiro-Wilk em cada grupo, obtendo $W=0.9374, p$-value $=0.3508$ e $W=0.9168, p$-value $=0.172$, o que garante suas normalidades. $O$ teste $F$ foi utilizado para inferir a variância dos grupos, obtendo $F=0.7586$, num $d f=14$, denomd $f=14, p$-value $=0.6123$, o que garantiu com 95\% de confiança que ambos os grupos possuem a mesma variância, possibilitando a aplicação do teste de hipótese $T$ para comparar as médias dois grupos. A médiado grupo1 foi 4.733333 e desvio padrão 1.498412, enquanto a média do grupo 2 foi 6.066667 com desvio padrão de 1.720327 . O resultado do teste $t(t=-2.2635, d f=28, p$ value $=0.03155)$ rejeitou a hipótese $h 0$ e comprovou com $95 \%$ de confiança que a média dos aprendizes, que não havia feito curso de inglês anteriormente e que realizou o experimento utilizando a ferramenta adaptativa, é maior e a diferença entre as médias, considerada significante estatisticamente.

Além dos resultados quantitativos dos aprendizes, também foi analisado qualitativamente o uso das ferramentas, Tabela 3. A linha 1 da Tabela 3 mostra o resultado referente a questão de usabilidade da ferramenta, onde a grande maioria (94\%) considerou fácil a utilização. Desse total, $46 \%$ concordou fortemente e $48 \%$ concordou com a facilidade de uso. A linha 2 relata a opinião dos aprendizes a respeito dos conteúdos apresentados, sendo $93 \%$ os que concordaram ou concordaram fortemente que o conteúdo é útil, claro e fácil de entender. Na linha 3 encontra-se o resultado sobre o tamanho do questionário utilizado para obtenção do estilo de aprendizagem e que foi proposto com o objetivo de comparar com o experimento anterior. A maioria dos aprendizes (56\%) não concordou com a afirmação de que o questionário era muito grande. Sendo $39 \%$ neutros, $13 \%$ os que discordaram e $4 \%$ os que discordaram fortemente. A linha 4 mostra o resultado sobre o entendimento das questões no questionário, onde $86 \%$ afirmou concordar ou concordar fortemente. A linha 5 relata a opinião dos aprendizes do grupo 2 a respeito do perfil do aluno para adaptação do curso. A maioria dos participantes $(82 \%)$ concordam com a afirmação que foi excelente a 
personalização por perfil e $88 \%$ acredita que tal fato contribuiu positivamente para seu aprendizado, linha 6. Por fim, a linha 7 mostra os resultados referentes a aceitação da ferramenta, onde $86 \%$ afirmou ter interesse em aprender outros assuntos com a mesma.

Tabela 3. Resultados Qualitativos.

\begin{tabular}{|c|c|c|c|c|c|}
\hline \multirow{2}{*}{ Pergunta } & \multicolumn{5}{|c|}{ Respostas } \\
\cline { 2 - 6 } & $\begin{array}{c}\text { Concordo } \\
\text { Fortemente }\end{array}$ & Concordo & Neutro & Discordo & $\begin{array}{c}\text { Discordo } \\
\text { Fortemente }\end{array}$ \\
\hline 1 & $46 \%$ & $48 \%$ & $6 \%$ & - & - \\
\hline 2 & $54 \%$ & $39 \%$ & $5 \%$ & $2 \%$ & - \\
\hline 3 & $11 \%$ & $33 \%$ & $39 \%$ & $13 \%$ & $4 \%$ \\
\hline 4 & $34 \%$ & $52 \%$ & $7 \%$ & $4 \%$ & $4 \%$ \\
\hline 5 & $38 \%$ & $44 \%$ & $16 \%$ & $3 \%$ & - \\
\hline 6 & $47 \%$ & $41 \%$ & $9 \%$ & $3 \%$ & - \\
\hline 7 & $38 \%$ & $43 \%$ & $16 \%$ & $4 \%$ & - \\
\hline
\end{tabular}

\section{Conclusão e Trabalhos Futuros}

Este trabalho teve como objetivo propor um novo modelo de usuário que faz uso de Estilos de Aprendizagem, Teoria da Resposta ao Item e Lógica Fuzzy, a fim de adaptar o conteúdo às características do aprendiz utilizando um sistema hipermídia adaptativo. Os resultados obtidos mostram-se promissores, tanto quantitativamente quanto qualitativamente, considerando que a grande maioria dos participantes gostou da adaptação do conteúdo ao seu EA e gostaria de fazer outros treinamentos adaptativos, além do grupo que utilizou a ferramenta adaptativa obter resultados superiores ao grupo de controle.

Em trabalhos futuros pretende-se aplicar o modelo em cursos de maior extensão de conteúdo, com o intuito de verificar a possível melhora na qualidade da modelagem do usuário devido ao grande volume de interações com o sistema. Também seria válido implementar um agente afetivo que possa acompanhar o aprendiz durante seus estudos, mantendo sua motivação e podendo dar feedbacks e dicas das dificuldades encontradas pelo aluno. Assim como sistemas multiagentes para educação, os agentes afetivos também são fontes de forte pesquisa [Frozza et al., 2007]. E por fim, testar o modelo proposto com outros questionários de identificação de estilos de aprendizagem, como por exemplo o teste de Ross [Ross \& Ross, 1976] e os questionários propostos por [Sonwalkar, 2001]. 


\section{Referências}

Andrade, D. F. De, Tavares, H. R., \& Valle, R. da C. (2000). Teoria da Resposta ao Item : Conceitos e Aplicações. Associação Brasileira de Estatística.

Baker, F. B. (2001). The Basics of Item Response Theory. (C. Boston \& L. Rudner, Eds.)ERIC Clearinghouse on Assessment and Evaluation (p. 180). Heinemann. doi:10.1111/j.1365-2362.2010.02362.x

Brasil, C. G. da I. no. (2012). Pesquisa sobre o uso das tecnologias de informação e comunicação no Brasil : TIC Educação 2011. (A. F. Barbosa, Ed.) (p. 400). São Paulo. Retrieved from http://op.ceptro.br/cgi-bin/cetic/tic-educacao-2011.pdf

Brusilovsky, P. (2001). Adaptive Hypermedia. User Modeling and UserAdapted Interaction, 11(1), 87-110. doi:10.1023/A:1011143116306

Brusilovsky, P., \& Nejdl, W. (2003). Adaptive hypermedia and adaptive web. In M. P. Singh (Ed.), Practical Handbook of Internet Computing. CRC Press. Retrieved from http://www.kbs.uni-hannover.de/Arbeiten/Publikationen/2003/brusilovskynejdl.pdf

Bueno, A. M. F., Brito, A. G. S., \& Brito, L. C. (2011). Abordagem Hipermídia Educacional Adaptativa para a Personalização do Processo de Ensino Através da Web. In XXII SBIE - XVII WIE (pp. 110-119).

Dias, C. C. L., Gasparini, I., \& Kemczinski, A. (2009). Identificação dos estilos cognitivos de aprendizagem através da interação em um Ambiente EAD. In XVII Workshop sobre Educação em Computa ção, XXIX Congresso da Sociedade Brasileira de Computação (pp. 489-498). Bento Gonçalves.

Felder, R. M., \& Silverman, L. K. (1988). Learning and Teaching Styles in Engeneering Education. Journal of Engineering Education, 78(June), 674-681. Retrieved from http://www.researchgate.net/publication/236261199_Comparative_Education_Tea cher_Training_Education_Policy_School_Leadership_and_Social_Inclusion/file/6 0b7d5176c814cd615.pdf\#page $=407$

Fleming, N. (2012). Introduction to Vark. Retrieved July 01, 2013, from http://legacy.hazard.kctcs.edu/VARK/introduction.htm

Frozza, R., Konzen, A., Mainieri, A. G., Schreiber, J., Molz, K., Tautz, J., ... Dresch, J. (2007). Agentes tutor e companheiro em um ambiente educacional baseado em estilos cognitivos.

Graf, S., Viola, S., \& Kinshuk, T. (2006). Representative characteristics of FelderSilverman learning styles: An empirical model. ... Cognition and Exploratory Learning in .... Retrieved from http://www.academia.edu/download/30950650/c52_papadimitriou_komis_tselios_ abouris_designing-pda-mediated-educational2006.pdf\#page $=254$ 
Kavitha, R., Vijaya, a., \& Saraswathi, D. (2012). Intelligent item assigning for classified learners in ITS using Item Response Theory and Point Biserial Correlation. 2012 International Conference on Computer Communication and Informatics, 1-5. doi:10.1109/ICCCI.2012.6158813

Kolb, D. (1974). On management and the learning process. Cambridge, Mass.: M.I.T. Retrieved from http://psycnet.apa.org/psycinfo/1974-33290-011

Mohamed, H., Bensebaa, T., \& Trigano, P. (2012). Developing Adaptive Intelligent Tutoring System based on Item Response Theory and Metrics. International Journal of ..., 43, 1-14. Retrieved from http://www.sersc.org/journals/IJAST/vol43/1.pdf

Pasquali, L., \& Primi, R. (2003). Fundamentos da Teoria da Resposta ao Item - TRI. Avaliação Psicológica, (2), 99-110.

Pedrycz, W. (1993). Fuzzy Control and Fuzzy Systems (p. Research Studies Press Ltd.Taunton\Somerset). John Wiley \& Sons. Retrieved from http://portal.acm.org/citation.cfm?id=529624

Ross, J. D., \& Ross, C. M. (1976). Teste Ross de Processos Cognitivos. Instituto Pieron de Psicologia Aplicada.

Sandri, S. (1999). Lógica Nebulosa. In Escola De Redes Neurais Conselho Nacional De Redes Neurais (pp. c073-c090). ITA.

Schnitman, I. M. (2010). O perfil do aluno virtual e as teorias de estilos de aprendizagem. In $3^{\circ}$ Simpósio Hipertexto e Tecnologias na Educação: redes sociais e aprendizagem (Vol. 1, pp. 1-10).

Silva, A. do S. da. (2000). TUTA - Um Tutor Baseado em Agentes no Contexto do Ensino a Distância. UFPB.

Sonwalkar, N. (2001). Changing the Interface of Education with Revolutionary Learning Technologies. Campus Technology. iUniverseBooks.

Spenassato, D., \& Kinas, P. G. (2010). Teoria da Resposta ao Item : estimação dos parâmetros pelos métodos de Amostragem por Importância Adaptativa e Monte Carlo via Cadeias de Markov. In CNMAC (pp. 1004-1005).

Valaski, J., Malucelli, A., \& Reinehr, S. (2011). Revisão dos Modelos de Estilos de Aprendizagem Aplicados à Adaptação e Personalização dos Materiais de Aprendizagem. Simpósio Brasileiro de Informática na Educação, 2-5. Retrieved from http://www.br-ie.org/pub/index.php/sbie/article/view/1843

Zadeh, L. A. (1965). Fuzzy sets. Information and Control, 8(3), 338-353. doi:10.1016/S0019-9958(65)90241-X 